\title{
THE CONTINUOUS LEGENDRE TRANSFORM, ITS INVERSE TRANSFORM, AND APPLICATIONS
}

\author{
P.L. BUTZER, R.L. STENS and M. WEHRENS \\ Lehrstuhl A für Mathematik \\ Aachen University of Technology \\ 51 Aachen, Western Germany \\ (Received January 15, 1979)
}

ABSTRACT. This paper is concerned with the continuous Legendre transform, derived from the classical discrete Legendre transform by replacing the Legendre polynomial $P_{k}(x)$ by the function $P_{\lambda}(x)$ with $\lambda$ real. Another approach to T.M. MacRobert's inversion formula is found; for this purpose an inverse Legendre transform, mapping $L^{1}\left(\mathbb{R}^{+}\right)$into $L^{2}(-1,1)$, is defined. Its inversion in turn is naturally achieved by the continuous Legendre transform. One application is devoted to the Shannon sampling theorem in the Legendre frame together with a new type of error estimate. The other deals with a new representation of Legendre functions giving information about their behaviour near the point $\mathrm{x}=-1$.

KEY WOROS AND PHRASES. Continuous Legendre Transform, Inverse Legendre Trans form, Inversion Formulae, Shannon Sampling Theorem, Truncation Error Estimates, Behaviour of Legendre Functions Near Singular Points.

AMS (MOS) SUBJECT CLASSIFICATION (1970) CODES. $44 \mathrm{~A} 15,33 \mathrm{~A} \mathrm{45,} 94 \mathrm{~A} 05$, 41 A 25. 


\section{INTRODUCTION}

In the early fifties C.J. Tranter (15) and R.V. Churchill (7) examined in some detail the (discrete) Legendre transform, which is defined in terms of the Legendre polynomials $P_{k}(x), k \in \mathbb{P}=\{0,1,2, \ldots\}$, and discussed its applicability mainly to the solution of partial differential equations. The present authors dealt with applications of this transform to a variety of problems in approximation theory in (14), (4), (5).

The first aim of this paper is to generalize this transform by replacing $\mathrm{k}$ by an arbitrary real $\lambda$, thus to study the transform

$$
f^{\wedge}(\lambda):=\frac{1}{2} \int_{-1}^{1} f(x) P_{\lambda}(x) d x,
$$

$\left\{\mathrm{P}_{\lambda}(\mathrm{x}) ; \lambda \in \mathbb{R}\right\}$ being the system of Legendre functions. It will be referred to as the first continuous Legendre transform of $f$.

of basic importance in such a study is the existence of a further transform leading to an inversion formula, enabling one to reconstruct the original function $f$ from the values $f^{\wedge}(\lambda)$ of $f^{\wedge}$. T.M. MacRobert found such a formula in $(10)$, and gave sufficient conditions for its validity in (11). The latter result was recalled with another proof by L. Robin (12, pp. 131). The transform in question is defined by

$$
\hat{F} F(x):=4 \int_{0}^{\infty} F(\lambda) P_{\lambda-1 / 2}(-x) \lambda \sin \pi \lambda d \lambda \quad(x \in(-1,1)),(1.2)
$$

$F$ being defined on $\mathbb{R}^{+}=[0, \infty)$, and it leads under certain conditions to the inversion formula

$$
\wedge\left[f^{\wedge}(\cdot-1 / 2)\right](x)=f(x)
$$

This inverse transform will be called the second continuous Legendre transform of F. Our first objective will be to prove the inversion under conditions different to those of MacRobert and Robin (Theorem 1). 
Given the transform (1.2), there also arises the question as to its inversion. One naturally expects that the transform leading to it is (1.1). It will be shown that this is indeed so for a special class of functions, with

$$
\left[{ }^{\wedge} F\right]^{\wedge}(\lambda-1 / 2)=F(\lambda) \quad\left(\lambda \in \mathbf{R}^{+}\right) .
$$

This result, which does not seem to have been considered previously, is dealt with in Theorem 2 .

Two applications will be considered. The first is concerned with a version of the Shannon sampling theorem in the Legendre setting which is more constructive than that of L.L. Campbell (6). Legendre transform methods will also enable us to present a new type of truncation error estimate for the corresponding sampling sum. The second application deals with a representation of the Legendre function $P_{\lambda}(x)$ as a sum of an infinite series of Legendre polynomials and an extra term describing the singularity of $P_{\lambda}(x)$ at the point $x=-1$.

\section{PRELIMINARIES}

The Legendre functions will be defined by means of the hypergeometric series. Let $a, b, c$ be real numbers, $c \neq 0,-1,-2, \ldots$, and $(a)_{0}:=1,(a)_{k}:=a(a+1) \ldots(a+k-1)$, $k \in \mathbb{N}$. The hypergeometric series

$$
F(a, b ; c ; x):=\sum_{k=0}^{\infty} \frac{(a)_{k}(b)_{k}}{(c)_{k} k !} x^{k}
$$

is absolutely and uniformly convergent on each compact subinterval of $(-1,1)$. The series also converges for $x=-1$ if $c-a-b>-1$, and for $x=1$ provided that $c-a-b>0$. In the latter case

$$
\lim _{x \rightarrow 1-} F(a, b ; c ; x)=F(a, b ; c ; 1)=\frac{\Gamma(c) \Gamma(c-a-b)}{\Gamma(c-a) \Gamma(c-b)}
$$

provided the right-hand side is meaningful. (For these properties see e.g. W.N. Bailey (1).)

The Legendre functions $P_{\lambda}(x)$ are now defined for $x \in(-1,1], \lambda \in \mathbb{R}$ by 


$$
P_{\lambda}(x):=F\left(-\lambda, \lambda+1 ; 1 ; \frac{1-x}{2}\right) \equiv \sum_{k=0}^{\infty} \frac{(-\lambda)_{k}(\lambda+1)_{k}}{(k !)^{2}}\left(\frac{1-x}{2}\right)^{k} .
$$

Since $P_{-\lambda}(x)=P_{\lambda-1}(x)$, it suffices to consider the case $\lambda \geqslant-1 / 2$ in the following. The $P_{\lambda}$ are arbitrarily often differentiable on $(-1,1]$, and they satisfy

$$
\begin{array}{lr}
\frac{d}{d x}\left[\left(x^{2}-1\right) P_{\lambda}^{\prime}(x)\right]=\lambda(\lambda+1) P_{\lambda}(x) & (x \in(-1,1] ; \lambda \geqslant-1 / 2),(2.4) \\
\left(x^{2}-1\right) P_{\lambda}^{\prime}(x)=\lambda\left(x P_{\lambda}(x)-P_{\lambda-1}(x)\right) & (x \in(-1,1] ; \lambda \geqslant-1 / 2),(2.5) \\
P_{\lambda}(1)=1, \quad P_{\lambda}^{\prime}(1)=\lambda(\lambda+1) / 2 & (\lambda \geqslant-1 / 2) .(2.6)
\end{array}
$$

Since for each $\lambda \geqslant-1 / 2$ there exists a constant $M_{\lambda}$ such that

$$
\left|\frac{(-\lambda)_{k}(\lambda+1)_{k}}{(k !)^{2}}\right| \leqslant M_{\lambda} k^{-1} \quad(k \in \mathbb{N})
$$

it follows that

$$
\begin{aligned}
& \left|P_{\lambda}(x)\right| \leqslant 1+M_{\lambda} \sum_{k=1}^{\infty} \frac{1}{k}\left(\frac{1-x}{2}\right)^{k} \\
& =1+M_{\lambda} \log \frac{2}{1+x}
\end{aligned}
$$$$
(x \in(-1,1] ; \lambda \geqslant-1 / 2) .(2 \cdot 7)
$$

This implies that for each $\lambda \geqslant-1 / 2$

$$
\begin{gathered}
P_{\lambda} \in L^{p}(-1,1) \\
\lim _{x \rightarrow(-1)+}(1+x) P_{\lambda}(x)=0 .
\end{gathered}
$$

Here $L^{p}(-1,1), 1 \leqslant p<\infty$, denotes the space of all real-valued functions $f$ defined on $(-1,1)$ endowed with the norm

$$
\|f\|_{p}:=\left\{\frac{1}{2} \int_{-1}^{1}|f(x)|^{p} d x\right\}^{1 / p} .
$$


A relation of type (2.8) is needed for the derivative $\mathrm{P}_{\lambda}^{\prime}(\mathbf{x})$. For this purpose one has by $(2.5)$ and $(2.3)$ for $\lambda \notin \mathbb{P}$

$$
\begin{aligned}
& \left(x^{2}-1\right) P_{\lambda}^{\prime}(x)-\lambda(x+1) P_{\lambda}(x)=-\lambda\left(P_{\lambda}(x)+P_{\lambda-1}(x)\right) \\
& =-2 \lambda \sum_{k=0}^{\infty} \frac{(-\lambda)_{k}(\lambda)_{k}}{(k !)^{2}}\left(\frac{1-x}{2}\right)^{k}=-2 \lambda F\left(-\lambda, \lambda ; 1 ; \frac{1-x}{2}\right) .
\end{aligned}
$$

This yields by (2.2) and (2.8) that

$$
\lim _{x \rightarrow(-1)+}(1+x) P_{\lambda}^{\prime}(x)=\frac{1}{\Gamma(1+\lambda) \Gamma(1-\lambda)} .
$$

Therefore

$$
\lim _{x \rightarrow(-1)+}(1+x) P_{\lambda}^{\prime}(x)=\frac{\sin \pi \lambda}{\pi}
$$

this being trivial for $\lambda \in \mathbb{P}$. This enables us to prove

LEMMA 1. Let $\lambda, v \geqslant-1 / 2, \lambda \neq \nu, \lambda \neq-v-1$. Then

$$
\frac{1}{2} \int_{-1}^{1} P_{\lambda}(x) P_{\nu}(-x) d x=\frac{\sin \pi \lambda-\sin \pi \nu}{\pi(\lambda-\nu)(\lambda+\nu+1)} .
$$

PROOF. First note that the integral is absolutely convergent since $P_{\lambda} \in L^{2}(-1,1)$. One deduces by partial integration, using $(2.4),(2.9)$, and $(2.6)$, that

$$
\begin{aligned}
& \lambda(\lambda+1) \int_{-1}^{1} P_{\lambda}(x) P_{\nu}(-x) d x \\
& =\left.\left(x^{2}-1\right) P_{\lambda}^{\prime}(x) P_{\nu}(-x)\right|_{(-1)+} ^{1-}-\int_{-1}^{1}\left(x^{2}-1\right) P_{\lambda}^{\prime}(x) \frac{d}{d x} P_{\nu}(-x) d x \\
& =\frac{2}{\pi} \sin \pi \lambda+\left.\left(x^{2}-1\right) P_{\lambda}(x) P_{\nu}^{\prime}(-x)\right|_{(-1)+} ^{1-}+\int_{-1}^{1} P_{\lambda}(x) \frac{d}{d x}\left[\left(x^{2}-1\right) \frac{d}{d x} P_{\nu}(-x)\right] d x \\
& =\frac{2}{\pi} \sin \pi \lambda-\frac{2}{\pi} \sin \pi \nu+v(v+1) \int_{-1}^{1} P_{\lambda}(x) P_{\nu}(-x) d x .
\end{aligned}
$$


This gives $(2.10)$.

At this stage some elementary properties of the Legendre polynomials are needed (cf. (14)). In the particular case that $\lambda=n \in \mathbb{P}$ the series in (2.3) is finite, so that $P_{n}(x)$ reduces to a polynomial of degree $n$, the classical Legendre polynomial of $n$th degree in view of (2.4) and (2.6). These polynomials form an orthogonal set on $[-1,1]$, i.e., for $m, n \in \mathbb{P}$,

$$
\frac{1}{2} \int_{-1}^{1} P_{n}(x) P_{m}(x) d x= \begin{cases}1 /(2 n+1), & n=m \\ 0, & n \neq m .\end{cases}
$$

If $f \in X, X$ denoting one of the spaces $C[-1,1]$ or $L^{p}(-1,1), 1 \leqslant p<\infty$, the (discrete) Legendre transform is defined by

$$
f^{\wedge}(k)=\frac{1}{2} \int_{-1}^{1} f(x) P_{k}(x) d x
$$

it is a bounded, linear mapping from $X$ into the space $c_{0}$ of all null sequences. There holds the uniqueness theorem

$$
f^{\wedge}(k)=0 \quad(k \in \mathbb{P}) \leftrightarrow f(x)=0 \quad \text { (a.e. ), }
$$

and to each $f \in X$ one may associate its Legendre series

$$
f(x) \sim \sum_{k=0}^{\infty}(2 k+1) f^{\wedge}(k) P_{k}(x) .
$$

This series, which can be interpreted as the discrete counterpart of the inverse transform (1.2), does generally not represent the function $f$, but for $f \in L^{2}(-1,1)$ there holds

$$
\lim _{n \rightarrow \infty}\left\|\sum_{k=0}^{n}(2 k+1) f^{\wedge}(k) P_{k}(x)-f(x)\right\|_{2}=0 .
$$

of importance is the Parseval equality

$$
\frac{1}{2} \int_{-1}^{1} f(x) g(x) d x=\sum_{k=0}^{\infty}(2 k+1) f^{\wedge}(k) g^{\wedge}(k)
$$


valid for $f, g \in L^{2}(-1,1)$, and the fact that

$$
\left\|f-S_{n} f\right\|_{2}=E_{n}(f)
$$

Here $S_{n} f$ denotes the $n$th partial sum of the series in $(2.14)$, and $E_{n}(f)$ is the best approximation to $f$ by algebraic polynomials $p_{n}$ of degree $n$ in $L^{2}(-1,1)-$ -space, i.e.,

$$
E_{n}(f)=\inf _{p_{n}}\left\|f-p_{n}\right\|_{2} \text {. }
$$

Let us now return to the Legendre functions. Noting that $P_{k}(x)=(-1)^{k} P_{k}(-x)$ for $\lambda=k \in \mathbb{P}$, it follows from $(2.10)$ and (2.11) that

$$
\mathrm{P}_{\lambda}^{\wedge}(\mathrm{k})= \begin{cases}\frac{(-1)^{\mathrm{k}} \sin \pi \lambda}{\pi(\lambda-\mathrm{k})(\lambda+\mathrm{k}+1)}, & \lambda \neq \mathrm{k} \\ 1 /(2 \mathrm{k}+1) & , \quad \lambda=\mathrm{k}\end{cases}
$$

for $k \in \mathbb{P}$. Since $P_{\lambda} \in L^{2}(-1,1)$, one has ba taking $f=g=P_{\lambda}$ in (2.15) that for $\lambda \geqslant-1 / 2$

$$
\left\|P_{\lambda}\right\|_{2}^{2}=\left\{\begin{array}{ll}
\sum_{k=0}^{\infty}(2 k+1)\left[\frac{\sin \pi \lambda}{\pi(\lambda-k)(\lambda+k+1)}\right]^{2}, & \lambda \notin \mathbb{P} \\
1 /(2 \lambda+1) & , \lambda \in \mathbb{P} .
\end{array} .\right.
$$

This formula enables us to deduce

LEMMA 2. a) For each compact interval $[a, b] \subset(-1,1)$ there holds

$$
\left|P_{\lambda}(x)\right|=O\left(\lambda^{-1 / 2}\right) \quad(x \in[a, b] ; \lambda \rightarrow \infty)
$$

b)

$$
\left\|P_{\lambda}\right\|_{1} \leqslant\left\|P_{\lambda}\right\|_{2}=O\left(\lambda^{-1 / 2}\right) \quad(\lambda \rightarrow \infty) .
$$

c) For each $[c, d] \subset[-1 / 2, \infty)$ there exists $M>0$ such that

$$
\left\|P_{\lambda}(x)-P_{\nu}(x)\right\|_{2} \leqslant M|\lambda-\nu| \quad(\lambda, \nu \in[c, d]) .
$$


PROOF. For a) see (8, formulae 3.9.1 (2) and 1.18 (5)). Concerning c), one has for $\lambda, v \notin \mathbb{P}$ for each $\mathbb{N} \in \mathbb{N}$ by $(2.18)$ and (2.15)

$$
\begin{aligned}
\left\|P_{\lambda}-P_{\nu}\right\|_{2}^{2} & =\sum_{k=0}^{\infty}(2 k+1)\left[\frac{\sin \pi \lambda}{\pi(\lambda-k)(\lambda+k+1)}-\frac{\sin \pi \nu}{\pi(\nu-k)(\nu+k+1)}\right]^{2} \\
& =\left(\sum_{k=0}^{N}+\sum_{k=N+1}^{\infty}\right)(2 k+1)\left(\int_{\lambda}^{\nu} q_{k}(x) d x\right)^{2}=: s_{1}+s_{2},
\end{aligned}
$$

where

$$
q_{k}(x):=\frac{(x-k)(x+k+1) \pi \cos \pi x-(2 x+1) \sin \pi x}{\pi(x-k)^{2}(x+k+1)^{2}} .
$$

Choosing $N \geqslant \max \{|a|,|b|\}$, yields

$$
\begin{gathered}
S_{1} \leqslant|v-\lambda|^{2} \sum_{k=0}^{N}(2 k+1) \sup _{x \in[a, b]}\left|q_{k}(x)\right|^{2}=M_{N}|\nu-\lambda|^{2}, \\
S_{2} \leqslant|\nu-\lambda|^{2} \sum_{k=N+1}^{\infty}(2 k+1)\left[\frac{(N+k)(N+k+1)+(2 N+1)}{(k-N)^{2}(k+1-N)^{2}}\right]^{2}=M_{N}^{*}|v-\lambda|^{2} .
\end{gathered}
$$

This gives (2.20) if $\lambda, \nu$ are not integers. If one (or both) of the reals $\lambda, \nu$ belong to $\mathbb{P}$, the same proof applies with obvious modifications. Part b) follows by similar arguments.

\section{THE FIRST CONTINUOUS IEGENDRE TRANSFORM}

The aim of this section is to study the continuous analogue of the discrete Legendre transform (2.12) and its properties, including an inversion formula. We restrict the matter to functions $f \in L^{2}(-1,1)$. For such $f$ the function

$$
f^{\wedge}(\lambda)=\frac{1}{2} \int_{-1}^{1} f(x) P_{\lambda}(x) d x
$$

is called the first (continuous) Legendre transform of $f$.

LEMMA 3. Let $f \in L^{2}(-1,1)$.

a) For all $p>2$ one has

$$
f^{\wedge}(\cdot-1 / 2) \in C_{0}\left(\mathbb{R}^{+}\right) \cap L^{p}\left(\mathbb{R}^{+}\right)
$$


b)

$$
\left|f^{\wedge}(\lambda)\right|=O\left(\lambda^{-1 / 2}\right)
$$

PROOF. One has by the Schwarz inequality

$$
\left|f^{\wedge}(\lambda)\right| \leqslant \frac{1}{2} \int_{-1}^{1}\left|f(x) P_{\lambda}(x)\right| d x \leqslant\|f\|_{2}\left\|P_{\lambda}\right\|_{2} .
$$

This gives assertion b) by La. 2 b). On the other hand, one has by La. 2 c) that for $\lambda, \nu \geqslant-1 / 2$

$$
\lim _{\lambda \rightarrow \nu}\left|f^{\wedge}(\lambda)-f^{\wedge}(\nu)\right| \leqslant \lim _{\lambda \rightarrow \nu}\|f\|_{2}\left\|P_{\lambda}-P_{\nu}\right\|_{2}=0
$$

So $f^{\wedge}$ is continuous on $[-1 / 2, \infty)$, indeed $f^{\wedge}(\cdot-1 / 2) \in C_{0}\left(\mathbb{R}^{+}\right)$. That $f^{\wedge}(\cdot-1 / 2) \in L^{p}\left(\mathbf{R}^{+}\right)$ for each $p>2$ follows from (3.2) and La. 2 b).

For the inversion formula two further results will be needed.

LEMMA 4. Let $F$ be a function defined on $[0, \infty)$ such that $\sqrt{\lambda} F(\lambda) \in L^{1}\left(\mathbb{R}^{+}\right)$. Then

$$
G(x):=\int_{0}^{\infty} F(\lambda) P_{\lambda-1 / 2}(-x) \lambda \sin \pi \lambda d x \quad(x \in(-1,1))
$$

belongs to $C(-1,1) \cap L^{2}(-1,1)$.

PROOF. The existence of the integral for all $x \in(-1,1)$ follows from La. $2 a)$. Moreover, the generalized Minkowski inequality and La. 2 b) give

$$
\|G\|_{2} \leqslant \int_{0}^{\infty}|F(\lambda) \lambda|\left\|P_{\lambda-1 / 2}\right\|_{2} \mathrm{~d} \lambda \leqslant \mathrm{M} \int_{0}^{\infty}|F(\lambda)| \lambda^{1 / 2} \mathrm{~d} \lambda<\infty,
$$

so that $G \in L^{2}(-1,1)$. Finally, for arbitrary $x \in(-1,1)$ there exists a $\delta>0$ such that $x \pm \delta \in(-1,1)$, and one has for all $|y| \leqslant \delta$

$$
|G(x+y)-G(x)| \leqslant \int_{0}^{\infty}|F(\lambda) \lambda|\left|P_{\lambda-1 / 2}(-x-y)-P_{\lambda-1 / 2}(-x)\right| d \lambda .
$$

By La. 2 a) this integrand is bounded for $|y| \leqslant \delta$ by

$$
|F(\lambda) \lambda| 2 M \lambda^{-1 / 2} \in L^{1}\left(\mathbb{R}^{+}\right)
$$

So $G \in C(-1,1)$ by Lebesgue's dominated convergence theorem. 
PROPOSITION 1. One has for all $\mathrm{x} \in(-1,1)$ and $\mathrm{k} \in \mathbb{P}$

$$
\mathrm{P}_{\mathrm{k}}(\mathrm{x})=4 \int_{0}^{\infty} \mathrm{P}_{\mathrm{k}}^{\wedge}(\lambda-1 / 2) \mathrm{P}_{\lambda-1 / 2}(-\mathrm{x}) \lambda \sin \pi \lambda \mathrm{d} \lambda .
$$

PROOF. One has by (2.18) for $\lambda \geqslant 0, \lambda \neq k+1 / 2$,

$$
\mathrm{P}_{\mathrm{k}}^{\hat{k}}(\lambda-1 / 2)=\mathrm{P}_{\lambda-1 / 2}^{\hat{n}}(\mathrm{k})=\frac{(-1)^{\mathrm{k}} \sin \pi(\lambda-1 / 2)}{\pi(\lambda-\mathrm{k}-1 / 2)(\lambda+\mathrm{k}+1 / 2)},
$$

so that $\sqrt{\lambda} \mathrm{P}_{\mathrm{k}} \hat{\mathrm{k}}(\lambda-1 / 2) \in \mathrm{L}^{1}\left(\mathbb{R}^{+}\right)$. Denoting the integral in (3.3) by $\mathrm{G}_{\mathrm{k}}(\mathrm{x})$, one has that $G_{k} \in C(-1,1) \cap L^{2}(-1,1)$ by La. 4. Let us now evaluate the discrete Legendre transform (2.12) of the $G_{k}$. One has by Fubini's theorem for $k, j \in \mathbb{P}$

$$
\begin{aligned}
& \frac{1}{2} \int_{-1}^{1} G_{k}(x) P_{j}(x) d x \\
& =4 \int_{0}^{\infty} \frac{(-1)^{k} \sin \pi(\lambda-1 / 2)}{\pi(\lambda-k-1 / 2)(\lambda+k+1 / 2)}\left[\frac{1}{2} \int_{-1}^{1} P_{\lambda-1 / 2}(-x) P_{j}(x) d x\right] \lambda \sin \pi \lambda d \lambda \\
& =\frac{4(-1)^{k}}{\pi^{2}} \int_{0}^{\infty} \frac{\sin \pi(\lambda-1 / 2)}{\lambda^{2}-(k+1 / 2)^{2}} \frac{\sin \pi(\lambda-1 / 2)}{\lambda^{2}-(j+1 / 2)^{2}} \lambda \sin \pi \lambda d \lambda=: I,
\end{aligned}
$$

where (2.10) was used to evaluate the integral in square brackets. Now an elementary calculation shows that

$$
I=\frac{(-1)^{k}}{4 \pi^{2}(k+j+1) i} \lim _{R \rightarrow \infty} \int_{-R}^{R}\left[g_{1}(\lambda)-g_{2}(\lambda)\right] d \lambda,
$$

where

$$
\begin{aligned}
& g_{1}(z):=\frac{e^{i \pi z}+e^{i 3 \pi z}}{(z-k-1 / 2)(z-j-1 / 2)} \\
& g_{2}(z):=\frac{e^{-i \pi z}+e^{-i 3 \pi z}}{(z-k-1 / 2)(z-j-1 / 2)}
\end{aligned}
$$

$(z \in \mathbb{C}, z \neq k+1 / 2, z \neq j+1 / 2)$.

Now the function $g_{1}(z)-g_{2}(z)$ has only removable singularities, so that the latter integral can be replaced by a contour integral $\int_{C_{R}^{-}}\left[g_{1}(z)-g_{2}(z)\right] d z$, where $\mathrm{C}_{\mathrm{R}}^{-}$denotes the lower semicircle with radius $\mathrm{R}$ around the origin. Denoting the 
corresponding upper semicircle and the whole circle by $C_{R}^{+}$and $C_{R}$, respectively, it is easy to show by Jordan's lemma that

$$
\lim _{R \rightarrow \infty} \int_{C_{R}^{+}} g_{1}(z) d z=\lim _{R \rightarrow \infty} \int_{C_{R}^{-}} g_{2}(z) d z=0,
$$

so that

$$
\lim _{R \rightarrow \infty} \int_{-R}^{R}\left[g_{1}(\lambda)-g_{2}(\lambda)\right] d \lambda=\lim _{R \rightarrow \infty} \int_{C_{R}} g_{1}(z) d z
$$

The latter integral, however, can be computed by the residue theorem, to give

$$
\int_{C_{R}} g_{1}(z) d z= \begin{cases}(-1)^{k} 4 \pi^{2} i, & j=k \\ 0, & j \neq k\end{cases}
$$

for $R>\max \{k, j\}+1 / 2$. This in turn implies by (3.5) and (2.11) that

$$
G_{k}^{\wedge}(j)=\left\{\begin{array}{ll}
1 /(2 k+1), & k=j \\
0, & k \neq j
\end{array}\right\}=P_{k}^{\wedge}(j) \quad(j, k \in \mathbb{P})
$$

The proof is now completed by the uniqueness theorem (2.13).

We can now prove the inversion formula for the transform defined in (3.1).

THEOREM 1. Let $f \in L^{2}(-1,1)$ be such that $\sqrt{\lambda} f^{\wedge}(\lambda-1 / 2) \in L^{1}\left(\mathbb{R}^{+}\right)$.

a) One has for almost all $x \in(-1,1)$

$$
4 \int_{0}^{\infty} f^{\wedge}(\lambda-1 / 2) P_{\lambda-1 / 2}(-x) \lambda \sin \pi \lambda d \lambda=f(x) .
$$

b) If $\mathrm{f}$ is additionally continuous on $(-1,1)$, then $(3.6)$ holds everywhere on $(-1,1)$.

PROOF. Denoting the left-hand side of (3.6) by $\mathrm{J}(\mathrm{x})$, it follows from La. 4 that $J \in C(-1,1) \cap L^{2}(-1,1)$. For the discrete Legendre transform of $J$ one has for $k \in \mathbb{P}$ 


$$
\begin{aligned}
\frac{1}{2} \int_{-1}^{1} J(x) P_{k}(x) d x & =4 \int_{0}^{\infty} f^{\wedge}(\lambda-1 / 2)\left[\frac{1}{2} \int_{-1}^{1} P_{\lambda-1 / 2}(-x) P_{k}(x) d x\right] \lambda \sin \pi \lambda d \lambda \\
& =4 \int_{0}^{\infty}\left[\frac{1}{2} \int_{-1}^{1} f(y) P_{\lambda-1 / 2}(y) d y\right]\left[P_{k}(-\cdot)\right]^{\wedge}(\lambda-1 / 2) \lambda \sin \pi \lambda d \lambda \\
& =\frac{1}{2} \int_{-1}^{1} f(y)(-1)^{k}\left[4 \int_{0}^{\infty} P_{k}^{\wedge}(\lambda-1 / 2) P_{\lambda-1 / 2}(y) \lambda \sin \pi \lambda d \lambda\right] d y,
\end{aligned}
$$

the interchange of the order of integration being justified by a double use of Fubini's theorem. Now the inner integral is equal to $\mathrm{P}_{k}(-\mathrm{y})=(-1)^{\mathrm{k}} \mathrm{P}_{\mathrm{k}}(\mathrm{y})$ by Prop. 1. This implies that $J^{\wedge}(k)=f^{\wedge}(k), k \in \mathbb{P}$, so that the proof of part a) follows by (2.13). Part b) follows since in this case both sides of (3.6) are continuous.

\section{THE SECOND CONTINUOUS LEGENDRE TRANSFORM}

In this section to a function $F$ defined on $\mathbb{R}^{+}$the integral

$$
4 \int_{0}^{\infty} F(\lambda) P_{\lambda-1 / 2}(-x) \lambda \sin \pi \lambda d \lambda \quad(x \in(-1,1)) \quad(4.1)
$$

will be associated. It will be shown that the first Legendre transform (3.1) of the integral (4.1) reproduces $F$ under certain assumptions upon F.

Let $F \in L^{1}\left(\mathbb{R}^{+}\right)$such that $\sqrt{\lambda} F(\lambda) \in L^{1}\left(\mathbb{R}^{+}\right)$. The integral (4.1) is called the second (continuous) Legendre transform of $F$, denoted by $\hat{\wedge}(x)$. It follows from La. 4 that $\hat{\wedge} F(x)$ exists for all $x \in(-1,1)$ and belongs to $C(-1,1) \cap L^{2}(-1,1)$. For this transform one has the following inversion formula.

THEOREM 2. Let $F \in L^{1}\left(\mathbb{R}^{+}\right)$such that $\sqrt{\lambda} F(\lambda) \in L^{1}\left(\mathbf{R}^{+}\right)$. Assume that for the Fourier-cosine transform $F_{c}[F]$ of $F$ there holds

$$
F_{c}[F](v):=\sqrt{\frac{2}{\pi}} \int_{0}^{\infty} F(\lambda) \cos v \lambda d \lambda=0 \quad(\pi \leqslant v<\infty) . \quad \text { (4.2) }
$$

Then one has for almost all $\lambda \in \mathbb{R}^{+}$

$$
\frac{1}{2} \int_{-1}^{1} \hat{F}(x) P_{\lambda-1 / 2}(x) d x=F(\lambda) \text {. }
$$


If moreover $\mathrm{F} \in \mathrm{C}\left(\mathbb{R}^{+}\right)$, then (4.3) holds for all $\lambda \in \mathbb{R}^{+}$.

PROOF. One has by Fubini's theorem and La. 1 that

$$
\begin{aligned}
\frac{1}{2} \int_{-1}^{1} \hat{A} F(x) P_{\lambda-1 / 2}(x) d x & =4 \int_{0}^{\infty} F(\sigma)\left[\frac{1}{2} \int_{-1}^{1} P_{\sigma-1 / 2}(-x) P_{\lambda-1 / 2}(x) d x\right] \sigma \sin \pi \sigma d \sigma \\
& =4 \int_{0}^{\infty} F(\sigma) h_{\sigma}(\lambda) \sigma \sin \pi \sigma d \sigma=: H(\lambda),
\end{aligned}
$$

where

$$
h_{\sigma}(\lambda):=\frac{\cos \pi \sigma-\cos \pi \lambda}{\pi\left(\lambda^{2}-\sigma^{2}\right)}
$$

Applying Fubini's theorem once more shows that $H \in L^{1}\left(\mathbb{R}^{+}\right)$and

$$
F_{c}[H](v)=4 \int_{0}^{\infty} F(\sigma) \sigma \sin \pi \sigma F_{c}\left[h_{\sigma}\right](v) d \sigma .
$$

To calculate the Fourier-cosine transform of $h_{\sigma}$ we proceed in a manner similar to the proof of Prop. 1. One has for $v \geqslant 0$

$$
\begin{aligned}
& F_{c}\left[h_{\sigma}\right](v)=\lim _{R \rightarrow \infty} \int_{-R}^{R}\left[j_{1}(\lambda)-j_{2}(\lambda)\right] d \lambda, \\
& j_{1}(z):=\frac{1}{4} \sqrt{\frac{2}{\pi}} \frac{2 e^{i v z} \cos \pi \sigma-e^{i z(v+\pi)}}{\pi\left(z^{2}-\sigma^{2}\right)} \\
& j_{2}(z):=\frac{1}{4} \sqrt{\frac{2}{\pi}} \frac{e^{i z(v-\pi)}}{\pi\left(z^{2}-\sigma^{2}\right)} . \\
& (z \in \mathbf{C} ; z \neq \pm \sigma)
\end{aligned}
$$

Replacing the latter integral by a contour integral along $\mathrm{C}_{\mathrm{R}}^{+}$yields

$$
\begin{aligned}
F_{c}\left[h_{\sigma}\right](v) & =-\lim _{R \rightarrow \infty} \int_{C_{R}^{+}}\left[j_{1}(z)-j_{2}(z)\right] d z \\
& =\lim _{R \rightarrow \infty} \int_{R} j_{2}^{+} j_{2}(z) d z
\end{aligned}
$$

since $\int_{C_{R}^{-}} j_{1}(z) d z$ vanishes for $R \rightarrow \infty$. If $v \geqslant \pi$, then the last integral in $(4.6)$ also 
vanishes for $R \rightarrow \infty$, so that $F_{c}\left[h_{\sigma}\right](v)=0$ for $\pi \leqslant v<\infty$. On the other hand, if $0 \leqslant v<\pi$, then $\lim _{R \rightarrow \infty} \int_{C_{R}^{-}} j_{2}(z) d z=0$, so that

$$
F_{c}\left[h_{\sigma}\right](v)=\lim _{R \rightarrow \infty} \int_{C_{R}} j_{2}(z) d z=\sqrt{\frac{2}{\pi}} \frac{1}{2 \sigma} \sin (\pi-v) \sigma \quad(0<v<\pi ; \sigma>0),
$$

the latter equality holding again by the residue theorem. So it follows by (4.5) that

$$
\begin{aligned}
F_{c}[H](v) & =2 \sqrt{\frac{2}{\pi}} \int_{0}^{\infty} F(\sigma) \sin \pi \sigma \sin (\pi-v) \sigma d \sigma \\
& =\sqrt{\frac{2}{\pi}} \int_{0}^{\infty} F(\sigma)(\cos v \sigma-\cos (2 \pi-v) \sigma) d \sigma . \\
& =F_{c}[F](v)-F_{c}[F](2 \pi-v)=F_{c}[F](v) \quad(0 \leqslant v<\pi) .
\end{aligned}
$$

Moreover, $F_{c}[H](v)=0$ for $\pi \leqslant v<\infty$. Since $F_{c}[F](v)=0$ for $\pi \leqslant v<\infty$ by assumption, one finally has $F_{c}[H](v)=F_{c}[F](v)$ for all $v \in \mathbb{R}^{+}$. This implies (4.3) for almost all $\lambda \in \mathbb{R}^{+}$by the uniqueness theorem for the Fourier-cosine transform. If $\mathrm{F} \in \mathrm{C}\left(\mathbb{R}^{+}\right)$, then (4.3) holds for all $\lambda \in \mathbb{R}^{+}$since the left-hand side of (4.3) defines a continuous function on $\mathbb{R}^{+}$in view of La. 4 and La. 3. This completes the proof.

The results of Theorems 1 and 2 show that the first and second Legendre transform are inverse to another. Indeed, if $f \in L^{2}(-1,1)$ satisfies the assumptions of Thm. 1, then

$$
\wedge\left[f^{\wedge}(\cdot-1 / 2)\right](x)=f(x)
$$

for almost all $x \in(-1,1)$. On the other hand, if $F \in L^{1}\left(\mathbf{R}^{+}\right)$satisfies the assumptions of Thm. 2, then, for almost all $\lambda \in \mathbf{R}^{+}$,

$$
[\wedge F]^{\wedge}(\lambda-1 / 2)=F(\lambda)
$$




\section{APPLICATIONS}

\subsection{THE SAMPLING THEOREM}

L.L. Campbell (6) stated a sampling theorem for functions $F$ which are representable as the first Legendre transform of $f \in L^{2}(-1,1)$. We add a simple proof of this result (Thm. 3) based on Parseval's formula (2.15) (for the analogue in the classical setting see e.g. (2), (13)). Combining this result with our Thm. 2 yields that a function $F$, band-limited in the sense of the Fourier-cosine transform, satisfies the hypotheses of this theorem. This will be shown in Cor. 1 . Some error estimates will also be given.

THEOREM 3. If $F \in C\left(\mathbf{R}^{+}\right)$has the representation

$$
F(\lambda)=\frac{1}{2} \int_{-1}^{1} f(x) P_{W \lambda-1 / 2}(x) d x \quad\left(\lambda \in R^{+}\right)
$$

for some $f \in L^{2}(-1,1)$ and $W>0$, then one has for all $\lambda \in \mathbb{R}^{+}$

$$
F(\lambda)=\sum_{k=0}^{\infty}(2 k+1) F\left(\frac{k+1 / 2}{W}\right) \frac{\sin \pi(W \lambda-(k+1 / 2))}{\pi\left[w^{2} \lambda^{2}-(k+1 / 2)^{2}\right]}
$$

the series being absolutely and uniformly convergent on $\mathbf{R}^{+}$.

PROOF. Using (5.1), Passeval's equality (2.15) and (2.18), we obtain

$$
\begin{gathered}
F(\lambda)=\sum_{k=0}^{\infty}(2 k+1) f^{\wedge}(k) P_{W \lambda-1 / 2}^{\wedge}(k) \\
=\sum_{k=0}^{\infty}(2 k+1) F\left(\frac{k+1 / 2}{W}\right) \frac{\sin \pi(W \lambda-(k+1 / 2))}{\pi\left[W^{2} \lambda^{2}-(k+1 / 2)^{2}\right]} .
\end{gathered}
$$

Now Thm. 2 enables one to give more constructive conditions upon F for (5.2) to be valid.

COROLLARY 1. Let $F \in L^{1}\left(\mathbb{R}^{+}\right) \cap C\left(\mathbb{R}^{+}\right)$such that $\sqrt{\lambda} F(\lambda) \in L^{1}\left(\mathbb{R}^{+}\right)$, and $F_{c}[F](v)=0$ for $v>\pi W$, some $W>0$. Then there holds $(5.2)$.

PROOF. It is easy to see that $F(\cdot / W)$ satisfies the conditions of Thm. 2. This implies 


$$
\begin{array}{ll}
F(\sigma / W)=[\wedge F(\cdot / W)]^{\wedge}(\sigma-1 / 2) & \left(\sigma \in \mathbb{R}^{+}\right), \\
F(\lambda)=[\wedge F(\cdot / W)]^{\wedge}(\lambda W-1 / 2) & \left(\lambda \in \mathbb{R}^{+}\right) .
\end{array}
$$

Since $\wedge F(\cdot / W) \in L^{2}(-1,1)$, the representation (5.1) holds, and the assertion follows by Thm. 3 .

Thm. 3 and Cor. 1 show that a continuous function $F$ on $\mathbb{R}^{+}$satisfying certain conditions can be represented by its sampling sum on $\mathbb{R}^{+}$. Denoting the $n$th partial sum of the series in $(5.2)$ by $\left(S_{W, n} F\right)(\lambda)$, one may ask for the error which occurs if one approximates $\mathrm{F}$ by $\mathrm{S}_{\mathrm{W}, \mathrm{n}} \mathrm{F}$. This leads to the following truncation error estimate.

LEMMA 5. For each $F$ satisfying the assumptions of Thm. 3 for some fixed $W>0$ there exists a constant $M>0$ such that

$$
\left|F(\lambda)-\left(S_{W, n} F\right)(\lambda)\right| \leqslant M(\lambda+1)^{-1 / 2} E_{n}(f) \quad(n \in \mathbb{N} ; \lambda \geqslant 0)
$$

where $f$ is defined via $(5.1)$.

PROOF. Denoting the $n$th partial sum of the Fourier-Legendre series (2.14) of $f$ by $S_{n} f$, one can rewrite the $S_{W, n} F$ in view of (2.15) as

$$
\begin{aligned}
\left(S_{W, n} F\right)(\lambda) & =\sum_{k=0}^{\infty}(2 k+1)\left[S_{n} f\right]^{\wedge}(k) P_{W \lambda-1 / 2}^{\hat{N}}(k) \\
& =\frac{1}{2} \int_{-1}^{1}\left(S_{n} f\right)(x) P_{W \lambda-1 / 2}(x) d x .
\end{aligned}
$$

This yields by Schwarz' inequality

$$
\begin{aligned}
& \left|F(\lambda)-\left(S_{W, n} F\right)(\lambda)\right| \leqslant \frac{1}{2} \int_{-1}^{1}\left|f(x)-\left(S_{n} f\right)(x)\right|\left|P_{W \lambda-1 / 2}(x)\right| d x \\
& \leqslant\left\|f-S_{n} f\right\|{ }_{2}\left\|P_{W \lambda-1 / 2}\right\|_{2}=E_{n}(f)\left\|P_{W \lambda-1 / 2}\right\|_{2},
\end{aligned}
$$


the latter equality holding by (2.16). The desired result now follows from La. 2 b).

The error estimate given by La. 5 does not seem to be known in the classical setting (cf. (9), (3)). The factor $(\lambda+1)^{-1 / 2}$ diminishes the error, especially for large $\lambda$. This is due to the fact that condition (5.1) implies that $F(\lambda)=$ $O\left(\lambda^{-1 / 2}\right)$ by La. $\left.3 \mathrm{~b}\right)$.

The foregoing lemma may be specialized.

LEMMA 6. Let $F \in C\left(\mathbb{R}^{+}\right)$satisfy (5.1) with $f \in L^{2}(-1,1)$. If there exists a function $F^{*} \in C\left(\mathbb{R}^{+}\right)$satisfying $(5.1)$ with $f^{*} \in L^{2}(-1,1)$ such that

$$
F^{*}\left(\frac{k+1 / 2}{W}\right)=k^{\alpha} F\left(\frac{k+1 / 2}{W}\right)
$$

for some $\alpha>0$, then one has for all $n \in \mathbb{N}, \lambda>0$

$$
\left|F(\lambda)-\left(S_{W, n} F\right)(\lambda)\right| \leqslant M(\lambda+1)^{-1 / 2} n^{-\alpha_{E}} E_{n}\left(f^{*}\right)
$$

PROOF. As in the proof of La. 5 one obtains by Parseval's formula (2.15)

$$
\begin{aligned}
& \left|F(\lambda)-\left(S_{W, n} F\right)(\lambda)\right| \leqslant\left\|f-S_{n} f\right\|_{2}\left\|P_{\lambda W-1 / 2}\right\|_{2} \\
& =\left\{\sum_{k=n+1}^{\infty}(2 k+1)\left|f^{\wedge}(k)\right|^{2}\right\}^{1 / 2}\left\|P_{\lambda W-1 / 2}\right\|_{2} .
\end{aligned}
$$

Using the additional fact that

$$
\left(f^{*}\right)^{\wedge}(k)=k^{\alpha} f^{\wedge}(k)
$$

in view of (5.4), the latter sum can be estimated by

$$
\begin{gathered}
\sum_{k=n+1}^{\infty}(2 k+1)\left|f^{\wedge}(k)\right|^{2}=\sum_{k=n+1}^{\infty}(2 k+1) k^{-2 \alpha}\left|f^{* \wedge}(k)\right|^{2} \\
\leqslant\left\{n^{-\alpha} E_{n}\left(f^{*}\right)\right\}^{2} .
\end{gathered}
$$

This implies (5.5) by La. 2 b). 
Note that Lemmas 5 and 6 may also be formulated for functions $F, F^{*}$ satisfying the assumptions of Cor. 1. Then $f$ and $f *$ in (5.3) and (5.5) have to be replaced by $\hat{A} F(\cdot / W)$ and $\wedge^{\wedge} *(\cdot / W)$, respectively.

Let us conclude this subsection by showing that Cor. 1 could also be deduced from the classical sampling theorem for band-limited functions (see e.g. (3), (13)). Indeed, the hypotheses of Cor. 1 on F imply those for the sampling theorem, so that the latter yields, when extending $F$ to the whole real axis $\mathbf{R}$ as an even function,

$$
\begin{aligned}
& F(\lambda)=F\left(\left(\lambda-\frac{1}{2 W}\right)+\frac{1}{2 W}\right)=\sum_{k=-\infty}^{\infty} F\left(\frac{k+1 / 2}{W}\right) \frac{\sin \pi(W \lambda-k-1 / 2)}{\pi(W \lambda-k-1 / 2)} \\
& =\lim _{n \rightarrow \infty}\left\{\sum_{k=0}^{n} F\left(\frac{k+1 / 2}{W}\right) \frac{\sin \pi(W \lambda-k-1 / 2)}{\pi(W \lambda-k-1 / 2)}+\sum_{k=0}^{n-1} F\left(\frac{k+1 / 2}{W}\right) \frac{\sin \pi(W \lambda+k+1 / 2)}{\pi(W \lambda+k+1 / 2)}\right\} \\
& =\lim _{n \rightarrow \infty}\left\{\sum_{k=0}^{n} F\left(\frac{k+1 / 2}{W}\right)(2 k+1) \frac{\sin \pi(W \lambda-k-1 / 2)}{\pi\left[W^{2} \lambda^{2}-(k+1 / 2)^{2}\right]}-F\left(\frac{n+1 / 2}{W}\right) \frac{\sin \pi(W \lambda+n+1 / 2)}{\pi(W \lambda+n+1 / 2)}\right\} \\
& =\lim _{n \rightarrow \infty}\left(S_{W, n} F\right)(\lambda),
\end{aligned}
$$

the last relation holding since $F(\lambda) \rightarrow 0$ for $\lambda \rightarrow \infty$ by La. 3 b).

\subsection{REPRESENTATION FORMULA FOR LEGENDRE FUNCTIONS}

Let us give another application of the theorems to a new representation of the Legendre functions $P_{\lambda}(x)$ describing in particular the behaviour of $P_{\lambda}(x)$ for $x \rightarrow(-1)+$. A first result of this type, basic for this paper, was the estimate (2.7). As will be seen, the $\log (2 /(1+x))$ term there is sharp.

LEMMA 7. For $h \in(-1,1)$ let

$$
\kappa(x ; h):=\left\{\begin{array}{cc}
{\left[\log \frac{2}{1+h}\right]^{-1}\left[\log \frac{1+x}{1-x}-\log \frac{1+h}{1-h}\right],} & h<x<1 \\
0 & ,-1<x<h
\end{array}\right.
$$


be the kernel of the integral means (see (14)). Then one has for $x, h \in(-1,1)$

$$
\begin{aligned}
& \kappa(\cdot ; h) \wedge(\lambda)=\left\{\begin{array}{cc}
{\left[\log \frac{2}{1+h}\right]^{-1} \frac{1-P_{\lambda}(h)}{\lambda(\lambda+1)},} & \lambda>-1 / 2, \lambda \neq 0 \\
1 \quad, \quad \lambda=0,
\end{array}\right. \\
& k(x ; h)=4 \int_{0}^{\infty} \frac{1-P_{\lambda-1 / 2}(h)}{\left(\lambda^{2}-1 / 4\right) \log (2 /(1+h))} P_{\lambda-1 / 2}(-x) \lambda \sin \pi \lambda d \lambda .
\end{aligned}
$$

The proof of (5.6) follows by integration by parts, noting (2.4) and (2.9), and assertion (5.7) by Thm. 1 .

This result enables one to deduce the desired representation formula. One has for $h \in(-1,1), \sigma \geqslant 0$ by Thm. 3

$$
\begin{aligned}
& \log \frac{2}{1+h} k(\cdot ; h)^{\wedge}(\sigma-1 / 2) \\
& =\frac{\sin \pi(\sigma-1 / 2)}{\pi\left(\sigma^{2}-1 / 4\right)} \log \frac{2}{1+h}+\sum_{k=1}^{\infty}(2 k+1) \frac{1-P_{k}(h)}{k(k+1)} \frac{\sin \pi(\sigma-1 / 2-k)}{\pi\left[\sigma^{2}-(k+1 / 2)^{2}\right]} .
\end{aligned}
$$

Replacing $\sigma$ by $\lambda+1 / 2$ gives for $\lambda \geqslant-1 / 2, \lambda \neq 0$,

$$
\frac{1-P_{\lambda}(h)}{\lambda(\lambda+1)}=\frac{\sin \pi \lambda}{\pi \lambda(\lambda+1)} \log \frac{2}{1+h}+\sum_{k=1}^{\infty}(2 k+1) \frac{1-P_{k}(h)}{k(k+1)} \frac{\sin \pi(\lambda-k)}{\pi(\lambda-k)(\lambda+k+1)},
$$

so that

$$
\begin{gathered}
P_{\lambda}(h)=1-\lambda(\lambda+1) \sum_{k=1}^{\infty} \frac{2 k+1}{k(k+1)} \frac{\sin \pi(\lambda-k)}{\pi(\lambda-k)(\lambda+k+1)} \\
+\lambda(\lambda+1) \sum_{k=1}^{\infty} \frac{2 k+1}{k(k+1)} \frac{\sin \pi(\lambda-k)}{\pi(\lambda-k)(\lambda+k+1)} P_{k}(h)-\frac{\sin \pi \lambda}{\pi} \log \frac{2}{1+h} .
\end{gathered}
$$

Now one can compute the Legendre transform $\mathrm{P}_{\lambda}^{\hat{\lambda}}(0)$ from the last formula, noting that the second series on the right is uniformly convergent with respect to $h$, and using (2.11), 


$$
\mathrm{P}_{\lambda}^{\wedge}(0)=1-\lambda(\lambda+1) \sum_{\mathrm{k}=1}^{\infty} \frac{2 \mathrm{k}+1}{\mathrm{k}(\mathrm{k}+1)} \frac{\sin \pi(\lambda-\mathrm{k})}{\pi(\lambda-\mathrm{k})(\lambda+\mathrm{k}+1)}-\frac{\sin \pi \lambda}{\pi} \frac{1}{2} \int_{-1}^{1} \log \frac{2}{1+\mathrm{h}} \mathrm{dh} .
$$

Since $\int_{-1}^{1} \log (2 /(1+h)) d h=2$, and

$$
\mathrm{P}_{\lambda}^{\wedge}(0)=\frac{\sin \pi \lambda}{\pi \lambda(\lambda+1)} \quad(\lambda \geqslant-1 / 2, \lambda \neq 0)
$$

by (2.18), it follows that

$1-\lambda(\lambda+1) \sum_{k=1}^{\infty} \frac{2 k+1}{k(k+1)} \frac{\sin \pi(\lambda-k)}{(\lambda-k)(\lambda+k+1)}-\frac{\sin \pi \lambda}{\pi}=\frac{\sin \pi \lambda}{\pi \lambda(\lambda+1)} \quad(\lambda \geqslant-1 / 2, \lambda \neq 0)$.

As an immediate consequence of (5.8) and (5.9) one therefore has

LEMMA 8. For $\lambda \geqslant-1 / 2, \lambda \neq 0$ there holds

$$
\begin{aligned}
P_{\lambda}(x)= & \lambda(\lambda+1) \sum_{k=1}^{\infty} \frac{2 k+1}{k(k+1)} \frac{(-1)^{k} \sin \pi \lambda}{\pi(\lambda-k)(\lambda+k+1)} P_{k}(x) \\
& +\frac{\sin \pi \lambda}{\pi}\left[1+\frac{1}{\lambda(\lambda+1)}-\log \frac{2}{1+x}\right] \quad(x \in(-1,1)) .
\end{aligned}
$$

The equality also holds for $\lambda=0$ if $(\sin \pi \lambda) / \pi \lambda(\lambda+1)$ is interpreted to be equal to 1 for $\lambda=0$.

This result shows in particular that $P_{\lambda}(x)$ can be written as

$$
\mathrm{P}_{\lambda}(x)=\widetilde{P}_{\lambda}(x)-\frac{\sin \pi \lambda}{\pi} \log \frac{2}{1+x} \quad(x \in(-1,1]),
$$

where $\widetilde{\mathrm{P}}_{\lambda}$ is continuous on the closed interval $[-1,1]$.

For another application of Thm. 1 see L. Robin (12, p. 131).

ACKNOWLEDGEMENT. The contribution of M. Wehrens was supported by DFG research grant Bu 166/27 which is gratefully appreciated. 


\section{REFERENCES}

1. Bailey, W.N. Generalized Hypergeometric Series, Hafner Publishing Company, New York, 1972.

2. Brown, J.I., Jr. Sampling theorem for finite energy signals, IEEE Trans. Information Theory IT-14 (1968), 818-819.

3. Butzer, P.L. and W. Splettstößer. Approximation und Interpolation durch verallgemeinerte Abtastsummen, Forsch.-ber. Nordrhein-Westfalen

$\mathrm{Nr}$. 2708, Westdeutscher Verlag, Opladen, 1977.

4. Butzer, P.L., R.L. Stens, and M. Wehrens. Approximation by algebraic convolution integrals. In: Approximation Theory and Functional Analysis (Proc. Conf. held at Campinas, Brazil, August 1977; Ed. J.B. Prolla), North-Holland Publishing Company, Amsterdam.

5. Butzer, P.L., R.L. Stens, and M. Wehrens. Saturation classes of the Cesàro and Abel-Poisson means of Fourier-Legendre series. Acta Math. Acad. Hungar. 33 (1978), 19-35.

6. Campbell, L.L. A comparison of the sampling theorems of Kramer and Whittaker. J. Soc. Indust. Appl. Math. 12 (1964), 117-130.

7. Churchill, R.V. The operational calculus of Legendre transforms, J. Math. and Phys. 33 (1954), 165-177.

8. Erdélyi, A., W. Magnus, F. Oberhettinger, and F.G. Tricomi. Higher Transcendental Functions, Vol. 1, McGraw-Hill Book Company, New York, 1953.

9. Jerri, A.J. The Shannon sampling theorem - its various extensions and applications: a tutorial review, Proc. IEEE 65 (1977), 1565-1596.

10. MacRobert, T.M. Fourier integrals, Proc. Roy. Soc. Edinburgh Sect. A 51 $(1931-1932), 116-126$.

11. MacRobert, T.M. Some integrals, with respect to their degrees, of associated Legendre functions, Proc. Roy. Soc. Edinburgh Sect. A 54 (1933-1934), $135-144$.

12. Robin, L. Fonctions sphériques de Legendre et fonctions sphéroidales, Tome 3, Gauthier-Villars, Paris, 1959.

13. Stens, R.L. Error estimates for sampling sums based on convolution integrals, (to appear).

14. Stens, R.L. and M. Wehrens. Legendre transform methods and best algebraic approximation, Comment. Math. Prace Mat. 22 (1979).

15. Tranter, C.J. Legendre transforms, Quart. J. Math. Oxford Ser. (2) 1 (1950), $1-8$. 


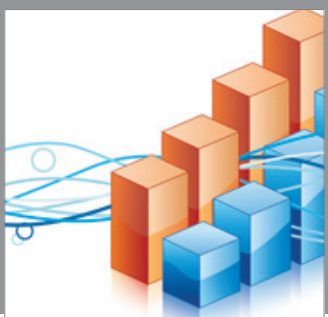

Advances in

Operations Research

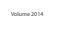

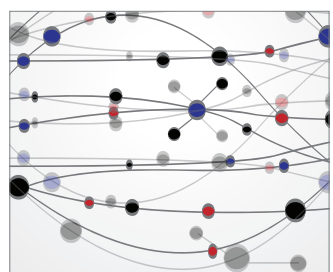

\section{The Scientific} World Journal
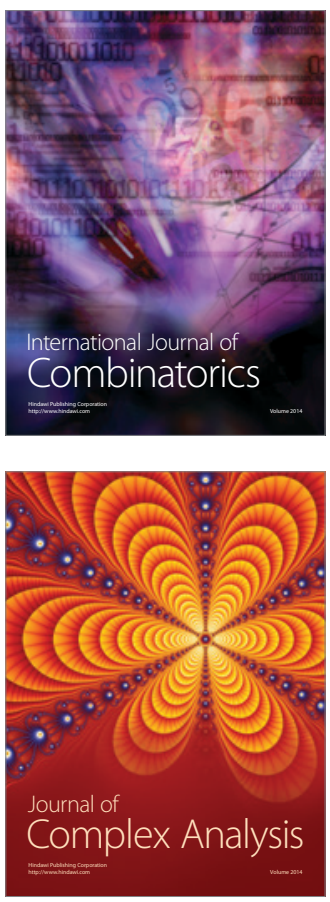

International Journal of

Mathematics and

Mathematical

Sciences
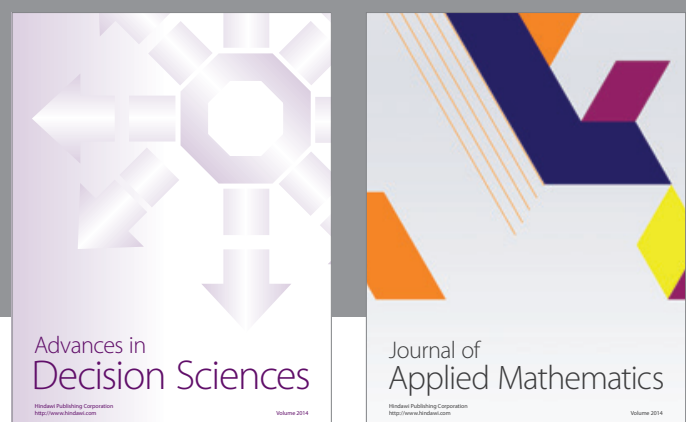

Journal of

Applied Mathematics
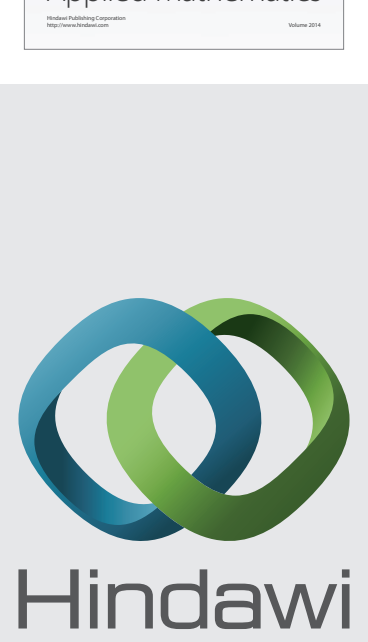

Submit your manuscripts at http://www.hindawi.com
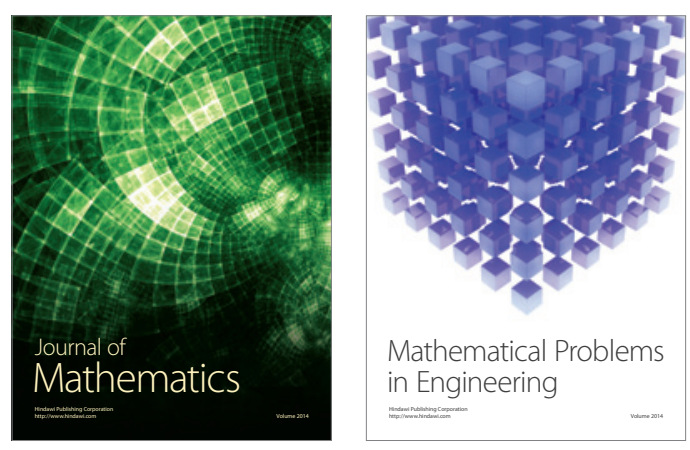

Mathematical Problems in Engineering
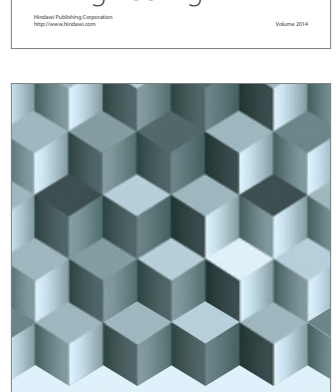

Journal of

Function Spaces
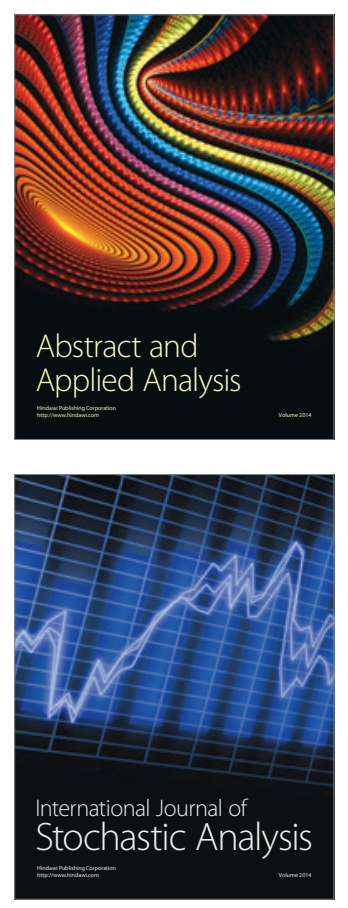

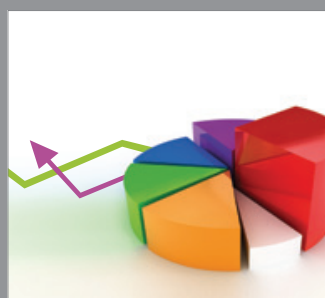

ournal of

Probability and Statistics

Promensencen
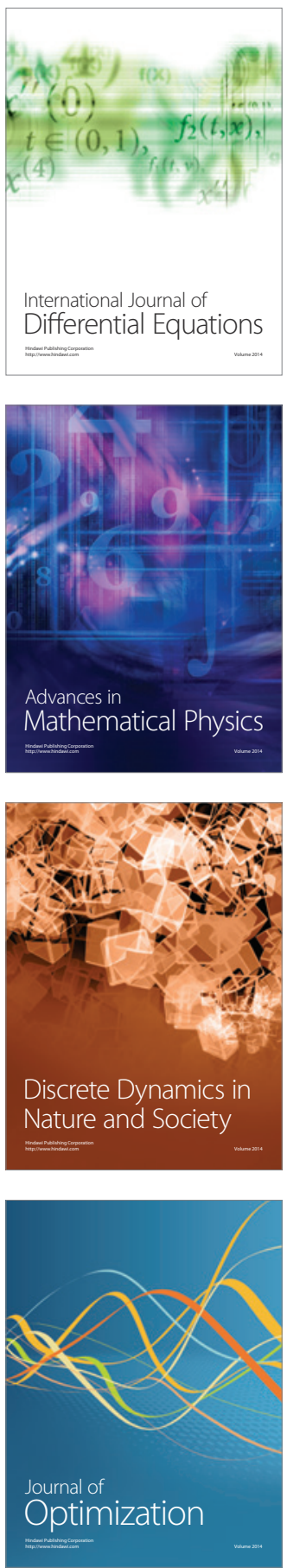